\title{
Modeling of cryo-adsorption of hydrogen on MOF-5 pellets: Effect of pellet properties on moderate pressure refueling
}

\author{
Jerome P. Ortmann \\ Chemical and Materials Systems Laboratory, \\ General Motors Global R\&D \\ Niket S. Kaisare \\ Department of Chemical Engineering, Indian Institute of Technology - Madras, \\ Chennai 600036, India
}

\section{Synopsis / Abbreviated Abstract}

The purpose of this research is to use a numerical model to study the influence of various physical parameters on refueling of a single MOF-5 pellet when exposed to hydrogen atmosphere at the final temperature and pressure; and therefore to design an optimal size of a single pellet that would meet the overall refueling targets for hydrogen cryo-adsorption.

For publication in International Journal of Hydrogen Energy 


\begin{abstract}
Refueling of a single MOF-5 pellet with cryogenic hydrogen at moderate pressures is modeled in this work. A MOF-5 pellet in a discharged state is placed in a hydrogen atmosphere at refueling conditions of $80 \mathrm{~K}$ temperature and 30 bar pressure. The absolute adsorption isotherm for hydrogen on MOF-5 is described by the Unilan model, hydrogen is modeled as a real gas, and properties of the compressed MOF-5 pellet are obtained from polynomial fits to low temperature experimental data. Pellet properties change due to the addition of expanded natural graphite (ENG) and various levels of compaction. A parametric study is performed using $\mathrm{COMSOL}^{\circledR}$ Multiphysics 4.2 to understand the effect of ENG and pellet compaction on refueling behavior of a single pellet. Specifically, the role of permeability and thermal conductivity, and the effect of anisotropic thermal conductivity are investigated using a two-dimensional axisymmetric model. The variation in pellet refueling time with varying pellet sizes is studied in order to determine the "optimal" pellet size for fast refueling. Results show that both stick-like geometry $(h / d \gg 1)$ and flat hockey-puck geometry $(h / d \ll 1)$ provide fast refueling. Conversely, pellets with $h / d \approx 0.5$ show the longest refueling times and are therefore not recommended for use in a packed bed design.
\end{abstract}




\section{INTRODUCTION}

There has been a renewed interest in the hydrogen economy [1] since the turn of this century due to higher efficiency and non-polluting products on using hydrogen to generate motive power $[2,3]$. Since hydrogen is not an energy source but an energy carrier, the hydrogen economy depends on efficient means to manufacture hydrogen. Another critical need to make the hydrogen economy feasible is to develop safe and inexpensive means to store and utilize hydrogen on-board a vehicle [4]. Storage of hydrogen in an adsorbent bed is being considered as a practical solution over other suggested strategies such as on-board generation or storage as a liquid or compressed gas [5-8].

Various methods of reversible hydrogen storage for vehicles may be classified as mechanical storage (e.g., compressed gas or liquid hydrogen), chemical storage (e.g., metal hydrides or imides) and adsorptive storage [9]. Adametz et al. [10] presented thermodynamic analysis of hydrogen storage on various adsorptive storage materials under cryogenic conditions. Several classes of materials show promising theoretical results; these include activated carbon-based materials [11], zeolites [12] and metal organic frameworks (MOFs) $[13,14]$. While these materials show promise, they currently do not meet the Department of Energy (DOE) targets [15] for on-board hydrogen storage [10]. The relevant DOE targets for 2017 are: $0.04 \mathrm{~kg}-\mathrm{H}_{2} / \mathrm{L}$-system volumetric storage capacity, $0.055 \mathrm{~kg}-\mathrm{H}_{2} / \mathrm{kg}$-system gravimetric capacity, 5 bar minimum delivery pressure and system refueling time of 3.3 minutes for $5 \mathrm{~kg}$ usable hydrogen [15].

Of specific interest in this work is the Metal Organic Framework, MOF-5, which is a microporous crystalline material consisting of tetrahedral $\mathrm{Zn}_{4} \mathrm{O}$ clusters coordinated with organic linkers to form three-dimensional periodic structures. The high gravimetric storage capacity of MOF-5 makes it an attractive candidate as an adsorbent material for hydrogen storage [6,1618]. The high gravimetric capacity is due to the highly porous structure of MOF-5 powder; though, this results in low volumetric capacity [19] and poor thermal properties. Benard et al. [18] demonstrated that MOF-5 itself would be unable to meet the US Department of Energy (DOE) targets [15] for on-board hydrogen storage. Hardy et al. [20] performed simulations on hydrogen cryogenic adsorption in a pressurized tank containing MaxSorb ${ }^{\mathrm{TM}}$ (activated carbon based material) and MOF-5. They concluded that although MOF-5 has lower volumetric storage capacity, the possibility of compacting MOF-5 to increase the storage capacity needs to be investigated [20].

MOF-5 pellets can be compacted to increase the volumetric storage capacity of hydrogen, although the gravimetric storage capacity decreases slightly. Purewal et al. [21] found that pellet 
compaction to $0.51 \mathrm{~g} / \mathrm{cm}^{3}$ (from the initial bulk density of $0.13 \mathrm{~g} / \mathrm{cm}^{3}$ ) provides a good compromise between the volumetric and gravimetric capacities. Expanded Natural Graphite (ENG) is added as an additive to improve the thermal properties of the pellets [22]. Compaction of the pellets also affects their permeability and effective thermal conductivity [22,24]. Liu et al. [22] showed that the addition of 5 to $10 \%$ ENG by weight improves thermal conductivity of compacted pellets, while resulting in only a modest decrease in hydrogen storage capacity. Due to the compaction process, the "worm-like" ENG is not randomly distributed, but aligns in plane of the cylindrical pellets. Consequently, the thermal conductivity of these pellets is anisotropic: the thermal conductivity in the radial direction is greater than that in the axial direction.

The aim of this work is to study the effect of various physical parameters of the MOF-5 pellet on its refueling time, analyze the role of anisotropic thermal conductivity and eventually find the optimal pellet geometry for fast refueling. Two-dimensional axisymmetric simulations of a single compacted MOF-5 pellet with varying amounts of ENG are conducted using $\mathrm{COMSOL}^{\circledR}$ finite element software.

Extensive simulations have been presented in the literature for adsorptive storage systems for cryogenic hydrogen storage. Senthil Kumar and Kumar [25] presented 1D simulations for flow-through refueling of tank containing MOF-5 adsorbent. They used real gas properties for hydrogen, but assumed the process to be isobaric. The latter assumption was relaxed in the 2D simulations of a MOF-5 bed presented by Kumar [26], though their use of excess adsorption and Langmuir isotherms limits the applicability of the model. Richard et al. [27] used a similar procedure for simulating refueling of an activated carbon (AX-21 and MaxSorb) fixed bed. They used the modified Dubinin-Astakhov adsorption model. Paggiaro et al. [28] used 2D simulations to demonstrate that conduction heat transfer through the $A X-21$ activated carbon bed could be the limiting factor for fast refueling or discharge of the AX-21 tank. Xiao et al. presented simulations of an activated carbon bed using both computational fluid dynamics employing Fluent [29] and the finite element method using $\operatorname{COMSOL}^{\circledR}[30,31]$.

In addition to the bed-level simulations, system-wide simulations have also been reported $[32,33]$. These are typically lumped parameter models and are executed using Simulink/ MATLAB. For example, Raju et al. [33] used their Simulink/MATLAB model for system-level simulations for complete FTP-75 and US06 drive cycles.

While past work has focused on thermodynamic analyses, simulations of refueling and discharge of the adsorbent bed, and system-wide simulations, the authors are not aware of any work targeting a single MOF-5 pellet. After the addition of ENG, the pellets are compacted into cylindrical shapes. Optimizing the pellet dimensions is important to ensure fast refueling. The 
model used herein is conceptually similar to the 2D fixed-bed simulations of Xiao et al. [30] and Senthil Kumar and Kumar [25], with the following changes. The model is re-written using the Unilan adsorption isotherm [23,34] in order to provide accurate fits with parameters having a clear physical interpretation for pellets containing either $5 \%$ or $10 \%$ ENG. The physical parameters are refitted to the range of pressure (up to 60 bars) and for newer experimental data available from DOE partners. The effect of thermal conductivity, accounting for the anisotropic conductivity (owing to ENG addition and pellet compaction), is investigated for the first time.

The paper is organized as follows. Section 2 describes the model used in this work; the Unilan adsorption isotherm is given in subsection 2.3 and physical properties of compacted MOF-5 pellets in subsection 2.4. Pellet refueling results are discussed in Section 3, and optimal sizing for cylindrical pellets are presented in Section 4, followed by conclusions in Section 5.

\section{MODEL DESCRIPTION}

Figure 1 shows a schematic of the cylindrical pellet of height $h$ and diameter $d$ modeled in this work. The aspect ratio is defined as $a r=h / d$. The nominal size of the pellet is considered to be $1 \mathrm{~cm}$ in height, with an aspect ratio of 1 . The pellet is initially under "discharged condition": the temperature inside the pellet is $140 \mathrm{~K}$, the pressure is 5 bar and the amount of hydrogen is equal to its equilibrium value as determined by the adsorption isotherm (discussed later in this section). At time $t=0$, the pellet is introduced into an atmosphere of hydrogen at $80 \mathrm{~K}$ and 30 bar pressure. The time evolution of temperature, pressure and adsorbed hydrogen profiles in the pellet is modeled. The model consists of material balance and energy balance for the pellet; steady state Darcy's law is used for momentum balance in the porous phase.

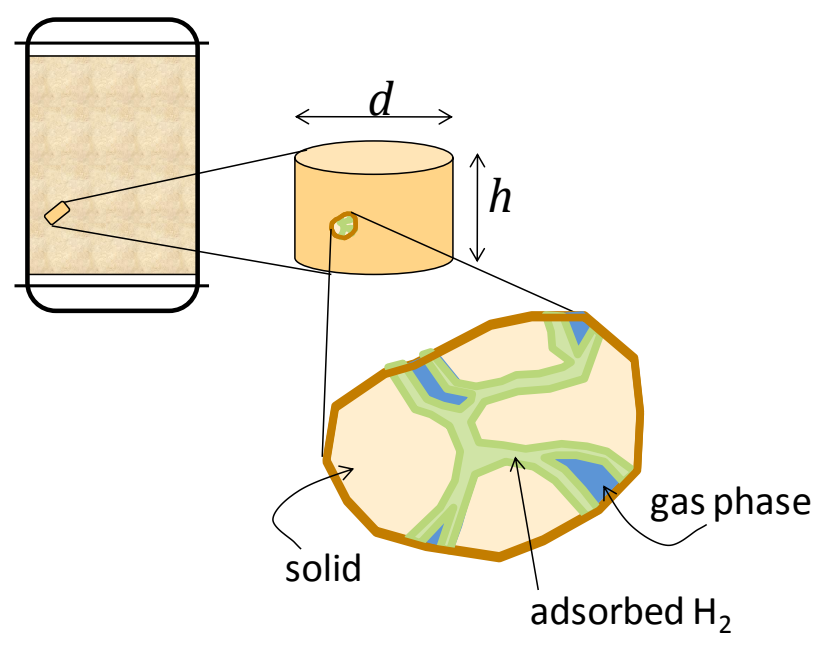

Figure 1: Schematic representation of the system. A single pellet in the adsorbent bed is being modeled. The zoomed in view of the control volume shows that hydrogen exists as 
gas phase (light blue color) and adsorbed phase (light green color). The solid and adsorbed hydrogen combined form the "condensed phase."

\subsection{GOVERNING EQUATIONS}

\subsubsection{Mass Balance}

The mass balance is written for the total amount of hydrogen in a control volume, which includes adsorbed hydrogen $\left(q \rho_{p} \mathrm{~kg} / \mathrm{m}^{3}\right.$, where $q$ is the absolute amount of adsorbed hydrogen in $\mathrm{kg}$ per kg-adsorbent) and gas phase hydrogen in the voids $\left(\varepsilon_{g} \rho_{g}\right)$.

$$
\frac{\partial}{\partial t}\left[q \rho_{p}+\varepsilon_{g} \rho_{g}\right]=-\nabla \cdot v \rho_{g}+\mathcal{D} \nabla^{2}\left(q \rho_{p}\right)
$$

Since the pellet size is of the order of $1 \mathrm{~cm}$, continuum approximation is used in developing the model used in this paper. In the above expression, the void fraction, $\varepsilon_{g}$ is calculated by subtracting the "adsorption volume" from the total void volume:

$$
\varepsilon_{g}=\varepsilon_{t o t}-\rho_{p} v_{a}
$$

where, $v_{a}$ is the adsorption volume in $\mathrm{m}^{3} / \mathrm{kg}$ of adsorbent. This value is obtained from the fits for the absolute adsorption isotherm. Since the total solid fraction of the pellet is the ratio of the density of the pellet to the structural density of MOF-5 adsorbent, we get

$$
\varepsilon_{\text {tot }}=1-\frac{\rho_{P}}{\rho_{s}}
$$

Thus, these parameters capture the effect of pore volume on hydrogen storage in the model. Besides pore volume, surface area also has a significant effect on hydrogen storage [35]. Both of these effects are captured through the Unilan adsorption isotherm, as described in Section 2.3.

The pellets are compacted to various levels [21], yielding densities in the range 0.3 to 0.79 $\mathrm{g} / \mathrm{cm}^{3}$. The thermal conductivity of a neat MOF- 5 pellet with a density of $0.5 \mathrm{~g} / \mathrm{cm}^{3}$ increases by a factor of 5 with the addition of $10 \%$ ENG [23]. A pellet density value of $\rho_{P}=0.5 \mathrm{~g} / \mathrm{cm}^{3}$ is used in this model because Purewal et al. [21] found that pellets of this density provide the best compromise between gravimetric and volumetric storage capacities. The structural density of MOF-5 is estimated to be $\rho_{S}=2030 \mathrm{~kg} / \mathrm{m}^{3}$.

The adsorption of hydrogen is assumed to follow the linear driving force model:

$$
\frac{d q}{d t}=k_{L D F}\left(q^{*}-q\right)
$$

where, $q^{*}(T, P)$ is the mass of hydrogen stored per unit mass of the adsorbent at equilibrium.

\subsubsection{Darcy's Law}

The relationship between the pressure drop and the superficial velocity in the porous pellet 
is given by Darcy's law:

$$
v=-\frac{\kappa}{\mu_{g}} \nabla P
$$

where, $\mu_{g}$ is viscosity of hydrogen and $\kappa$ is the permeability. As described in section 2.4.3, the value of permeability is obtained from experiments performed by Sudik and coworkers [24] at Ford Motor Company. Equations (1) and (3) are combined for the "transient Darcy's law" implementation in $\mathrm{COMSOL}^{\circledR}$.

\subsubsection{Energy Balance}

The energy balance needs to be carefully written for the system described by absolute adsorption of hydrogen on MOF-5. In the case of the excess adsorption based model of Kumar [26], it was natural to consider enthalpy balance, since the measured quantities include the excess adsorption isotherm and isosteric heat of adsorption. The absolute adsorption, on the other hand, lends itself naturally to internal energy balance.

As seen from the schematic in Figure 1, the control volume consists of condensed phase (adsorbent and adsorbed hydrogen) and gas phase hydrogen. As explained by Myers and Monson [36] and Richard et al. [37], the condensed phase may be assumed to be an ideal mixture of adsorbent and adsorbed hydrogen. The internal energy of the control volume is $\left(\rho_{P} U_{c}+\varepsilon_{g} \rho_{g} U_{g}\right) d V$; where $U_{c}$ and $U_{g}$ represent internal energy of the condensed phase and gas phase per unit mass, respectively.

The overall energy balance in descriptive form is written as:

$$
\frac{\partial}{\partial t}\left[\begin{array}{c}
\text { net energy } \\
\text { in control vol. }
\end{array}\right]+\nabla \cdot\left[\begin{array}{c}
\text { net flux } \\
\text { (convection) }
\end{array}\right]=\nabla \cdot\left[\begin{array}{c}
\text { net flux } \\
\text { (diffusive) }
\end{array}\right]-\nabla \cdot\left[\begin{array}{c}
\text { Pressure } \\
\text { effects }
\end{array}\right]+\left[\begin{array}{c}
\text { Viscous } \\
\text { source }
\end{array}\right]
$$

In the further derivation: the viscous effects will be neglected; diffusive flux is attributed to thermal conduction in the porous pellet, as well as the internal energy carried by the molecular diffusion of hydrogen; solid phase internal energy is assumed to be a function of only temperature (and not pressure); and hydrogen is treated as a real gas (no ideal gas assumption invoked).

Following the derivation presented in Refs. [25,30], the energy balance equation becomes:

$$
\left[\rho_{\mathrm{g}} c_{\mathrm{pg}}+\rho_{\mathrm{p}}\left(q c_{\mathrm{pg}}+c_{\mathrm{ps}}\right)\right] \frac{\partial T}{\partial t}+\rho_{\mathrm{g}} c_{\mathrm{p}, \mathrm{g}} \mathbf{v} \cdot \nabla T=\nabla \cdot\left(\lambda_{\mathrm{eff}} \nabla T\right)+\varepsilon_{\mathrm{g}} \alpha_{\mathrm{gP}} T \frac{\partial P}{\partial t}-\left(1-\alpha_{\mathrm{Pg}} T\right) \mathbf{v} \cdot \nabla P+\rho_{\mathrm{p}}\left(-\Delta h_{\mathrm{a}}\right) \frac{\partial q}{\partial t}
$$

The coefficient of the transient term represents the heat capacity of gaseous hydrogen and the condensed phase, respectively. The final term on the right hand side is the "heat" of adsorption. 


\subsection{INITIAL AND BOUNDARY CONDITIONS}

The pellet is assumed to be in a discharged condition at the initial time, with $T(0)=$ $140 \mathrm{~K}, P(0)=5 \mathrm{bar}$, and $q(0)=0.008$. Since the $2 \mathrm{D}$ model is axisymmetric, the symmetry boundary conditions are imposed at one of the boundaries. The pellet is introduced into a hydrogen atmosphere at the charging conditions. Thus, the Dirichlet boundary conditions of $T_{f}=80 \mathrm{~K}$ and $P_{f}=30 \mathrm{bar}$ are applied at all the remaining boundaries.

\subsection{ADSORPTION ISOTHERM}

The Unilan adsorption isotherm is used to describe the amount of hydrogen adsorbed at equilibrium per unit mass of the adsorbent, at a given temperature and pressure. This is represented by $\mathrm{q}^{*}$ in Eq. (2). Dundar et al. [34] reviewed the adsorption isotherms for hydrogen, and concluded that the Unilan model is appropriate for hydrogen adsorption on MOF-5. The Unilan isotherm is given by the following expression [23]:

$$
q^{*}=M_{H_{2}} * n_{a b s}=\frac{M_{H_{2}} n_{\max } R T}{E_{\max }-E_{\min }} \ln \left[\left(\frac{a+p \exp \left(E_{\max } / R T\right)}{a+p \exp \left(E_{\min } / R T\right)}\right)\right]
$$

where $a=\exp (-\Delta S / R)$. The excess adsorption data was generated by Sudik and coworkers at Ford Motor Company ${ }^{14,17}$ for MOF-5 pellets compacted to a density of $0.51 \mathrm{~g} / \mathrm{cm}^{3}$. The excess adsorption is related to absolute adsorption as:

$$
n_{e x}=n_{a b s}-\rho_{g} v_{a}=\left(\frac{q^{*}}{M_{H_{2}}}\right)-\rho_{g} v_{a}
$$

The Unilan model parameters obtained by Purewal et al. ${ }^{16}$ for MOF- 5 pellets are given in Table 1. The heat of adsorption is obtained from the Unilan isotherm $a^{16}$ :

$$
-\Delta H_{a}=\left.R T^{2} \frac{\partial \ln (P)}{\partial T}\right|_{q}=E_{\max }-\frac{(1-x) s}{1-\exp \left(\frac{(1-x) s}{R T}\right)}-\frac{x s}{1-\exp \left(\frac{-x S}{R T}\right)}
$$

where $s=E_{\max }-E_{\min }$ and $x=n_{a b s} / n_{\max }$.

\section{Table 1. Unilan Model Parameters for $\mathrm{H}_{2}$ Adsorption ${ }^{1}$}

$\begin{array}{ccccccc}\text { ENG \% } & \boldsymbol{\rho}\left(\mathbf{g} / \mathbf{c m}^{\mathbf{3}}\right) & \boldsymbol{\Delta S}(\mathbf{x R}) & \begin{array}{c}\boldsymbol{E}_{\max } \\ (\mathbf{k J} / \mathbf{m o l})\end{array} & \begin{array}{c}\boldsymbol{E}_{\min } \\ (\mathbf{k J} / \mathbf{m o l})\end{array} & \begin{array}{c}\boldsymbol{n}_{\max } \\ (\mathbf{m o l} / \mathbf{k g})\end{array} & \boldsymbol{v}_{\boldsymbol{a}}\left(\mathbf{c m}^{\mathbf{3}} / \mathbf{g}\right) \\ 5 & 0.47 & -7.88 & 4.76 & 2.11 & 41.3 & 0.95 \\ 10 & 0.48 & -7.86 & 4.96 & 1.55 & 44.6 & 1.08\end{array}$

The model developed in this work is applicable to other MOFs as well. The physical properties of porosity, adsorption volume and the adsorption isotherm parameters will vary for a different adsorbent material. This model can easily be extended if these properties are 
measured.

\subsection{PHYSICAL PROPERTIES OF MOF-5 PELLET}

\subsubsection{Specific Heat}

The following simplified polynomial expression was used for specific heat of MOF-5 pellet:

$$
c_{p, s}=\sum_{i=1}^{5} s_{i} T^{i-1}
$$

The overall fits listed in Table 2 are based on a compacted pellet, with a polynomial expression used for model stability.

\subsubsection{Thermal Conductivity}

Thermal conductivity measurements at cryogenic temperatures were obtained for various cases: neat MOF-5, MOF-5 with 5\% ENG and MOF-5 with 10\% ENG. Due to low thermal conductivity, neat MOF-5 pellets do not give acceptable refueling time. Therefore, the interest is in evaluating pellets with $5 \%$ and $10 \%$ ENG. Third order polynomials of the form

$$
\lambda_{a x}=\sum_{i=1}^{4} l_{i} T^{i-1}
$$

were fitted to the thermal conductivity data for MOF-5 pellets with $5 \%$ and $10 \%$ ENG. An intermediate thermal conductivity case is also considered in simulations, where the thermal conductivity is assumed to be twice that of the 5\% ENG case. The parameters for the $5 \%$ and 10\% ENG cases are given in Table 2.

Thermal conductivity of the compacted pellet is an important parameter affecting the refueling time for the pellet. During the compaction process (which is used to increase the pellet density), the ENG "worms" align along the plane of the cylindrical pellets. Thus, the in-plane thermal conductivity $\left(\lambda_{\text {radial }}\right)$ is up to 5 times greater than the axial thermal conductivity. The effect of anisotropic thermal conductivity is also investigated assuming a diagonal form of the thermal conductivity tensor:

$$
\Lambda=\left[\begin{array}{cc}
f_{r a d} \lambda_{a x} & 0 \\
0 & \lambda_{a x}
\end{array}\right]
$$

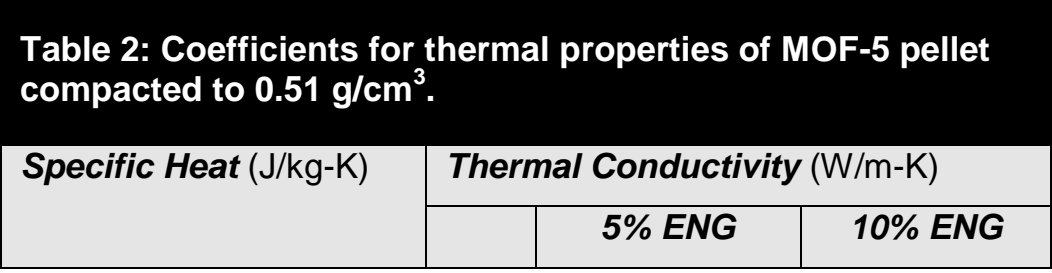




\begin{tabular}{|l|l|l|l|l|}
\hline$s_{1}$ & -49.950 & $l_{1}$ & $-2.268 \mathrm{e}-3$ & -0.26291 \\
\hline$s_{2}$ & 6.6724 & $l_{2}$ & $2.016 \mathrm{e}-3$ & 0.011744 \\
\hline$s_{3}$ & $-3.1196 \mathrm{e}-2$ & $l_{3}$ & $-5.696 \mathrm{e}-6$ & $-4.61 \mathrm{E}-05$ \\
\hline$s_{4}$ & $1.0533 \mathrm{e}-4$ & $l_{4}$ & 0.0 & $5.81 \mathrm{E}-08$ \\
\hline$s_{5}$ & $-1.3960 \mathrm{e}-7$ & & & \\
\hline
\end{tabular}

\subsubsection{Permeability}

Permeability in the porous medium depends on the diameter of individual MOF-5 particles, and the inter-particle void fraction:

$$
\kappa=\frac{\varepsilon_{\text {inter }}^{3} d_{p}^{2}}{150\left(1-\varepsilon_{\text {inter }}\right)^{2}}
$$

Since there is no direct measurement of either of these parameters, an estimated value of $\varepsilon=0.22$ is used. Permeability was measured for MOF-5 pellets at different compaction levels $\left(0.3 \mathrm{~g} / \mathrm{cm}^{3}\right.$ to $\left.0.5 \mathrm{~g} / \mathrm{cm}^{3}\right)$ [24]. Using Ergun's equation and assuming $\varepsilon=0.22$, the value of particle diameter was fitted to the data. Figure 2 shows the permeability values for various pellet densities. Based on these values, the sensitivity of the refueling time to pellet permeability is analyzed. Specifically, the following three values are considered: baseline value of $1.2 \times 10^{-14} \mathrm{~m}^{2}$, a high value of $2.1 \times 10^{-13} \mathrm{~m}^{2}$ (corresponding to $0.301 \mathrm{~g} / \mathrm{cm}^{3}$ MOF-5 pellet), and a low value of $5.1 \times 10^{-16} \mathrm{~m}^{2}$ (corresponding to $d_{p}=2 \mu \mathrm{m}$ ). This reasonably covers the range of permeability values observed in [24].

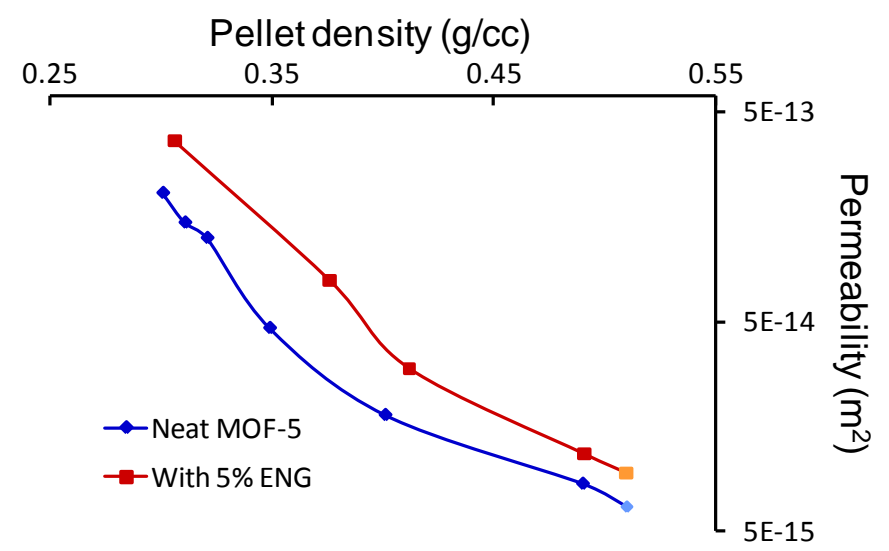

Figure 2: Variation in permeability with pellet density for neat MOF-5 pellets and pellets with $5 \%$ ENG. The last data point corresponding to $0.51 \mathrm{~g} / \mathrm{cm}^{3}$ pellet is extrapolated from the data. 


\subsection{PHYSICAL PROPERTIES OF HYDROGEN}

The physical properties of hydrogen were obtained from the NIST RefProps database $[38,39]$. The properties were expressed as functions of temperature and pressure. A polynomial was fitted to the compressibility data for a temperature range of 65 to $305 \mathrm{~K}$ and pressure range of 1 to 65 bar.

$$
Z(P, T)=1+B_{z}(T) P+C_{z}(T) P^{2}+D_{z}(T) P^{3}
$$

The density of hydrogen can then be calculated as:

$$
\rho_{g}=\frac{P M}{Z R T}
$$

Specific heat is often expressed as a polynomial of logarithm of temperature. However, such a form was found to be sensitive to parameter values. Hence, a polynomial form was used for specific heat as well:

$$
c_{p}=A_{c}(P)+\frac{B_{c}(P)}{T}+\frac{C_{c}(P)}{T^{2}}+D_{c}(P) T
$$

Finally, the Sutherland approximation was attempted for fitting the viscosity data. While the functional form was adequate for temperatures above $150 \mathrm{~K}$, some correction was required at lower temperatures. Thus, the following form was used:

$$
\mu_{g}=A_{\mu}(P)+B_{\mu}(P) T_{r}^{1 / 3}+\frac{C_{\mu}(P)}{T_{r}}
$$

\begin{tabular}{|c|c|c|}
\hline Compressibility (-) & Specific Heat (J/kg-K) & Viscosity (Pa-s) \\
\hline & $\begin{array}{cc}A_{c}(P)=a_{c 1}+ & a_{c 2} P+a_{c 3} P^{2}+a_{c 4} P^{3} \\
a_{c 1} & 1.9503 e 4 \\
a_{c 2} & -8.3051 \\
a_{c 3} & 2.8837 \\
a_{c 4} & -3.4213 e-2\end{array}$ & $\begin{array}{cc}A_{v}(P)=a_{v 1}+a_{v 2} P+a_{v 3} P^{2} \\
a_{v 1} & -9.6555 \mathrm{e}-6 \\
a_{v 2} & 1.1302 \mathrm{e}-8 \\
a_{v 3} & -6.7193 \mathrm{e}-10\end{array}$ \\
\hline $\begin{array}{cc}B_{z}(T)=b_{z 1} & +\frac{b_{z 2}}{T_{r}}+\frac{b_{z 3}}{T_{r}^{2}}+\frac{b_{z 4}}{T_{r}^{3}} \\
b_{z 1} & -1.1501 \mathrm{e}-4 \\
b_{z 2} & 9.2824 \mathrm{e}-3 \\
b_{z 3} & -2.5577 \mathrm{e}-2 \\
b_{z 4} & -1.0695 \mathrm{e}-2\end{array}$ & $\begin{array}{cc}B_{c}(P)=b_{c 1}+ & b_{c 2} P+b_{c 3} P^{2}+b_{c 4} P^{3} \\
b_{c 1} & -1.1655 \mathrm{e} 6 \\
b_{c 2} & 6.5414 \mathrm{e} 2 \\
b_{c 3} & -4.8813 \mathrm{e} 2 \\
b_{c 4} & 5.8287\end{array}$ & $\begin{array}{cc}B_{v}(P)=b_{v 1}+b_{v 2} P+b_{v 3} P^{2} \\
b_{v 1} & 8.7136 e-6 \\
b_{v 2} & -4.5954 e-9 \\
b_{v 3} & 2.7575 e-10\end{array}$ \\
\hline $\begin{array}{c}C_{z}(T)=c_{z 1}+\frac{c_{z 2}}{T_{r}}+\frac{c_{z 3}}{T_{r}^{2}}+\frac{c_{z 4}}{T_{r}^{3}} \\
c_{z 1} \\
4.4713 \mathrm{e}-6\end{array}$ & $\begin{array}{c}C_{c}(P)=c_{c 1}+c_{c 2} P+c_{c 3} P^{2}+c_{c 4} P^{3} \\
c_{c 1} \quad 3.9207 \mathrm{e} 7\end{array}$ & $\begin{array}{c}c_{v}(P)=c_{v 1}+c_{v 2} P+c_{v 3} P^{2} \\
c_{v 1}=3.6370 \mathrm{e}-6\end{array}$ \\
\hline
\end{tabular}

Table 3. Values of various parameters for calculating temperature and pressure dependent properties of pure hydrogen gas. Pressure units are bar and temperature K; reduced temperature $T_{r}=T / T_{c}$, where $T_{c}=33.145 \mathrm{~K}$. The various properties are in SI units. 


\begin{tabular}{|c|c|c|c|c|c|}
\hline $\begin{array}{l}c_{z 2} \\
c_{z 3} \\
c_{z 4}\end{array}$ & $\begin{array}{l}-7.2264 e-5 \\
3.4550 e-4 \\
-3.2069 e-4\end{array}$ & $\begin{array}{l}c_{c 2} \\
c_{c 3} \\
c_{c 4}\end{array}$ & $\begin{array}{l}3.2330 \mathrm{e} 5 \\
2.4894 \mathrm{e} 4 \\
-3.0842 \mathrm{e} 2\end{array}$ & $\begin{array}{l}c_{v 2} \\
c_{v 3}\end{array}$ & $\begin{array}{l}4.3738 e-9 \\
9.7752 e-10\end{array}$ \\
\hline $\begin{array}{r}D_{z}(T) \\
d_{z 1} \\
d_{z 2} \\
d_{z 3} \\
d_{z 4}\end{array}$ & $\begin{array}{l}+\frac{d_{z 2}}{T_{r}}+\frac{d_{z 3}}{T_{r}^{2}}+\frac{d_{z 4}}{T_{r}^{3}} \\
-6.4090 \mathrm{e}-8 \\
1.0079 \mathrm{e}-6 \\
-4.9093 \mathrm{e}-6 \\
7.7846 \mathrm{e}-6\end{array}$ & $\begin{array}{r}D_{c}(P)= \\
d_{c 1} \\
d_{c 2} \\
d_{c 3} \\
d_{c 4}\end{array}$ & $\begin{array}{l}d_{c 2} P+d_{c 3} P^{2}+d_{c 4} P^{3} \\
-5.7349 \\
1.8313 e-2 \\
-5.2438 e-3 \\
6.2135 e-5\end{array}$ & & \\
\hline
\end{tabular}

Table 3 lists the parameters and functional forms used to describe these properties. During linear regression, the minimum $R^{2}$ value of 0.995 was ensured. The maximum error (i.e., the infinite norm of the relative error) in the compressibility, specific heat and viscosity of hydrogen are $0.7 \%, 0.2 \%$ and $2.1 \%$, respectively.

\section{RESULTS AND DISCUSSIONS}

In this section, the effect of various design parameters on pellet refueling is analyzed. The baseline case refers to the parameter values described in section 2: Unilan isotherm parameters (see Table 1) and isotropic thermal conductivity corresponding to a MOF-5 pellet with $10 \%$ ENG, baseline permeability of $1.2 \times 10^{-14} \mathrm{~m}^{2}$, and pellet dimensions $h=d=1 \mathrm{~cm}$. Unless otherwise stated, the parameters are at their baseline values.

\subsection{PELLET REFUELING RESULTS}

Figure 3 shows the contours of weight percentage of hydrogen adsorbed (i.e., $q(t, r, z)$ in $\mathrm{kg}-\mathrm{H}_{2} / \mathrm{kg}$-adsorbent expressed as percentage) and temperature in the pellet at three different times during refueling. There is an initial steep rise in the amount of hydrogen adsorbed and the temperature in the pellet. For example, at 2 seconds (top-left panel of Figure 3), the center of the pellet has $1.2 \%$ hydrogen. The volume-averaged temperature and hydrogen concentration are shown as a blue solid line and a red dashed line, respectively, in Figure 4a. Starting with an initial adsorbed hydrogen amount of $0.8 \%$, the value rapidly increases within the initial 0.1 seconds to approximately 1.8 weight- $\% \mathrm{H}_{2}$. This is due to rapid pressurization of the pellet: The pressure contour at 0.1 seconds (Figure $4 \mathrm{~b}$ ) shows that the pressure within the entire pellet has reached within $1 \mathrm{kPa}$ (i.e., $0.01 \mathrm{bar}$ ) of the final pressure of $30 \mathrm{bar}$. Note that the deviation from 30 bar pressure is shown for easier viewing.

The rapid pressurization and heat released on adsorption results in an increase in the 
pellet temperature. During the transients, pellet temperature can exceed $160 \mathrm{~K}$ (bottom-left panel of Figure 3). Thus, the initial increase in the amount of hydrogen in the pellet is attributed to increased pressure from 5 to 30 bar. After this brief period of rapid pressurization accompanied by hydrogen adsorption, the amount of hydrogen stored depends primarily on the temperature. As the pellet cools from the periphery towards the center, the amount of hydrogen stored increases. It can be seen from the temporal plots of temperature and hydrogen content in the pellet that these contours follow the same trends.

Thus, the single pellet refueling occurs in two distinct phases: an initial phase of rapid pressurization accompanied by an increase in adsorbate concentration (and temperature), followed by gradual cooling of the pellet resulting in a further increase in adsorbate concentration due to favorable adsorption properties at lower temperature. The first phase will be affected by pellet permeability and initial pressure, whereas the second phase will be affected by pellet thermal dynamics. In both the cases, higher pellet surface area is conducive to faster refueling.
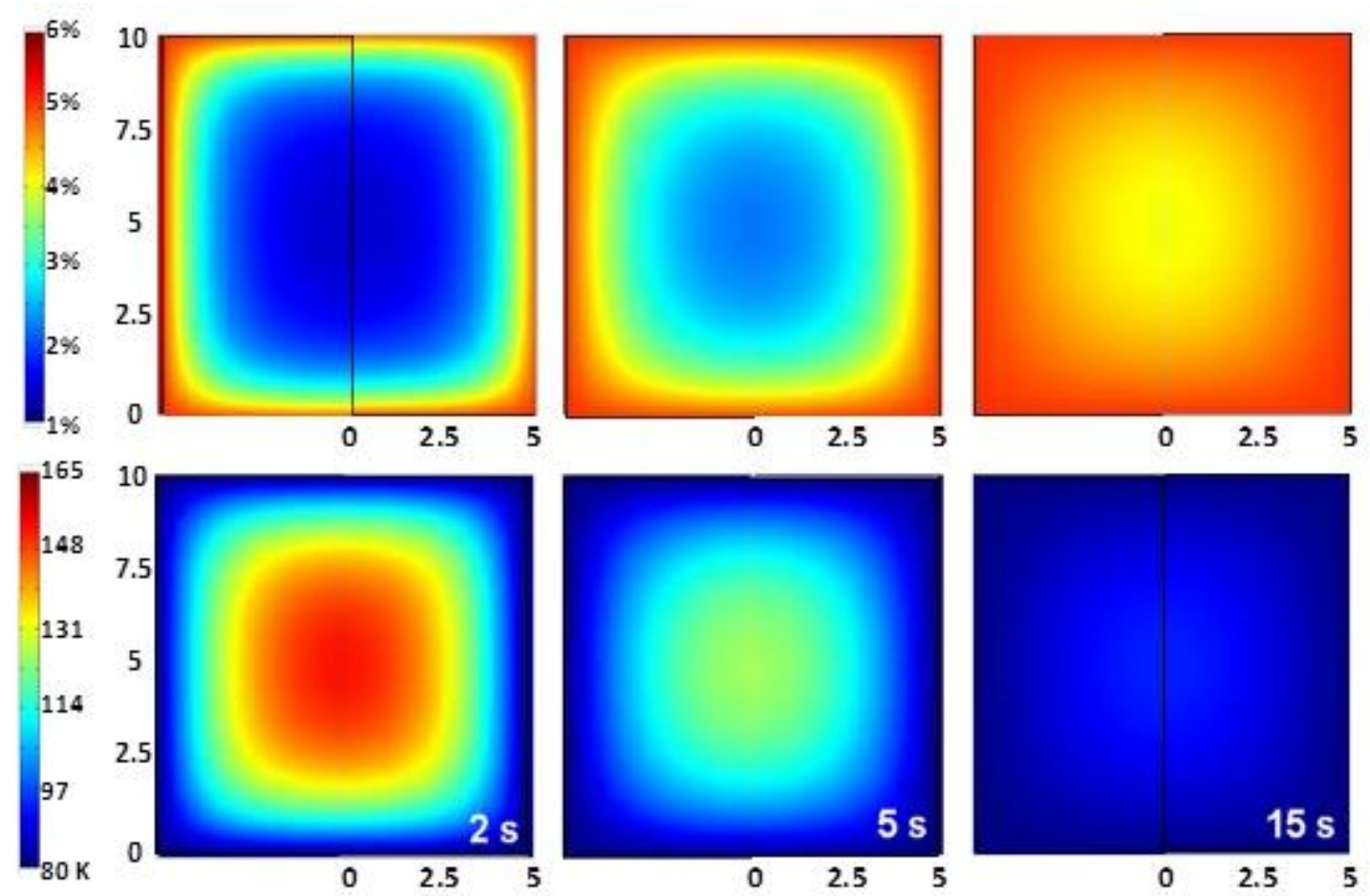

Figure 3: Amount of hydrogen stored in $\mathrm{kg}$ per $\mathrm{kg}$-adsorbent (top panels), and contours of pellet temperatures (bottom panels) at three different times for the nominal simulation conditions. Pellet dimensions are indicated in $\mathbf{~ m m}$. 

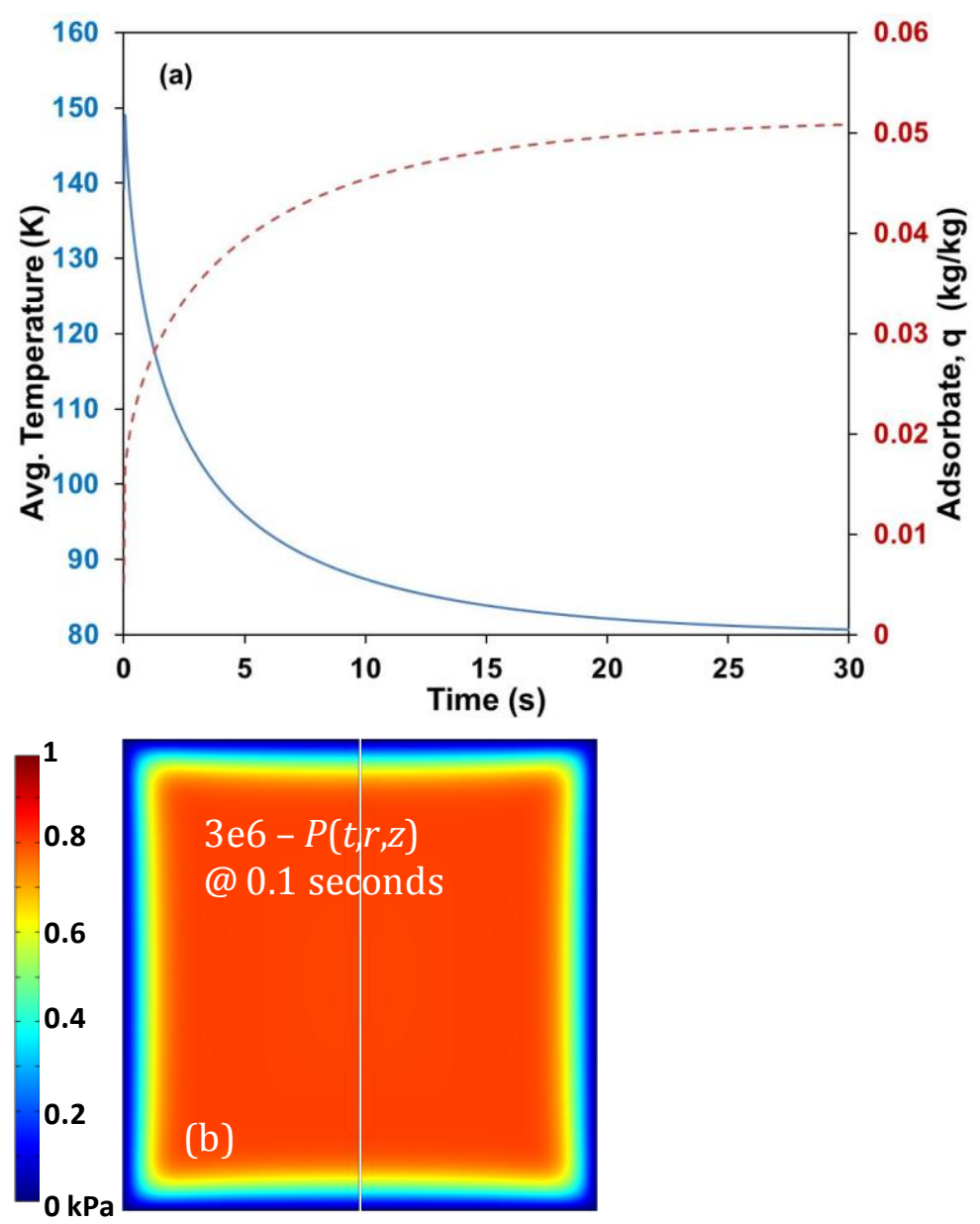

Figure 4: (a) Volume-averaged temperature and adsorbate amount vs. time; and (b) contours of pressure in the pellet at 0.1 seconds. In panel (b), the deviation of pressure from the final pressure (30 bar) is shown. Note that within 0.1 seconds, adsorbate concentration increases from 0.008 to $0.018 \mathrm{~kg}-\mathrm{H}_{2} / \mathrm{kg}$-adsorbent.

\subsection{EFFECT OF PERMEABILITY}

Figure 5 shows the contours of pressure and velocity for the three different values of pellet permeability, while Figure 6 shows the variation in the volume-averaged temperature of the pellet vs. refueling time for the three different permeability values. Only a single line is visible because all the three curves overlap. The pressure rapidly increases when the pellet, initially at 5 bar, is introduced in hydrogen at 30 bar pressure. The pressure and velocity contours are shown in Figure 5 at 0.1 seconds for all three values of permeability. In the figure, the deviation from 30 bar pressure is shown. The pressurization is rapid: the pressure tends to equilibrate at the speed of sound, whereas "resistance" from the porous medium slows down the equilibration 
time. Clearly, the time taken for pressure to equilibrate decreases as the permeability is increased. Nonetheless, even for a low value of permeability, the pressure equilibrates within 0.1 seconds.

The rapid pressurization, as well as heat released on adsorption, causes the pellet temperature to increase. The maximum increase in the local temperature, as expected, is highest for the low permeability case. The maximum local pellet temperature is $163.4 \mathrm{~K}, 164.6 \mathrm{~K}$ and $165.0 \mathrm{~K}$ for high, baseline, and low permeability cases.

These minor differences in the refueling behavior for the three values of permeability are not reflected in volume-averaged temperature (see Figure 6, where all three curves superimpose) and amount of hydrogen adsorbed (result not shown for brevity). This is because volume averaging smooths out the differences in the local profiles of temperature and adsorbed hydrogen.

In summary, pellet permeability does not have any impact on the refueling behavior of a single pellet with quiescent boundary conditions. These results may not be extrapolated to refueling of an adsorbent bed; pressure drop and flow rate through the bed may depend on the bed permeability.
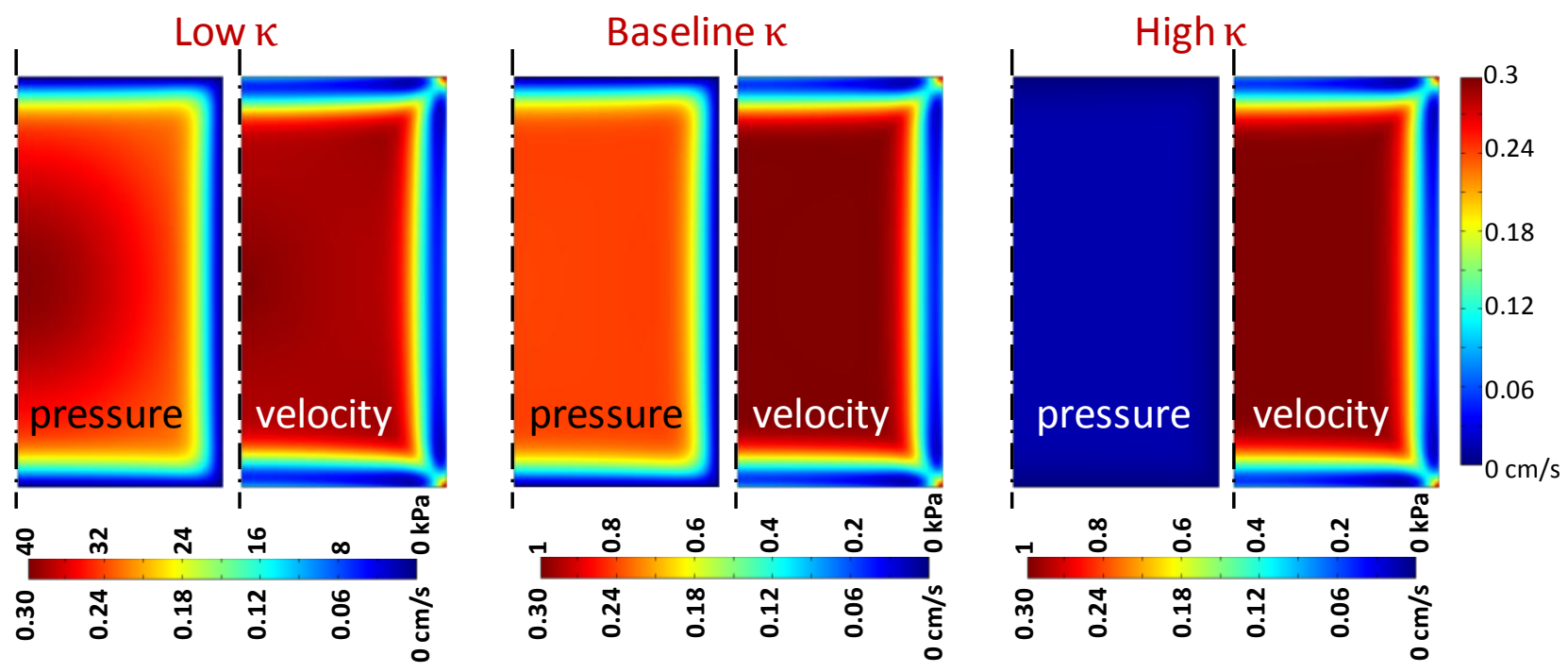

Figure 5: Contours of pressure and velocity at 0.1 seconds for the three values of permeability. In the pressure contours, the deviation from the final pressure of $\mathbf{3 0}$ bar $(3000 \mathrm{kPa})$ is shown. 


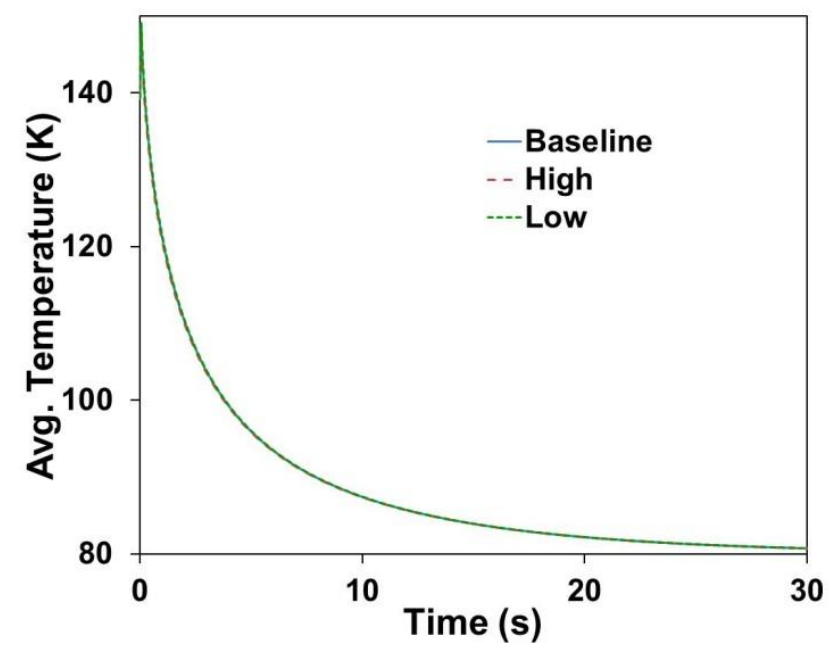

Figure 6: Volume-averaged temperature profile vs. time for the three values of permeability.

\subsection{EFFECT OF PELLET THERMAL CONDUCTIVITY}

Expanded natural graphite (ENG) is added to a MOF-5 pellet to improve its thermal and structural properties. Addition of ENG improves its thermal conductivity, while decreasing the total hydrogen storage capacity. Thus, there is a tradeoff in addition of ENG during pellet compaction. Here, the effect of pellet thermal conductivity is investigated. The Unilan parameters obtained for MOF-5 pellets with $5 \%$ and $10 \%$ ENG (see Table 1) are used.

Pellet thermal conductivity is one of the most important parameters that influence the rate of cooling, and therefore, the rate of pellet refueling. Three different cases of (isotropic) thermal conductivity are considered: compacted pellets with 10\% ENG, with 5\% ENG and an intermediate value of thermal conductivity. The low temperature thermal conductivity data was obtained from experiments by Sulic and coworkers at General Motors [40,41]; the parameters for the polynomial fits for the former two cases are given in Table 2. For the intermediate case, thermal conductivity is assumed to be twice the measured value at 5\% ENG. In all cases, the pellets have been compacted to a density of approximately $0.5 \mathrm{~g} / \mathrm{cm}^{3}$. For the sake of comparison, the thermal conductivity values for the three cases at $110 \mathrm{~K}$ are $0.55 \mathrm{~W} / \mathrm{m}-\mathrm{K}(10 \%$ ENG), $0.32 \mathrm{~W} / \mathrm{m}-\mathrm{K}$ (intermediate) and $0.16 \mathrm{~W} / \mathrm{m}-\mathrm{K}$ (5\% ENG).

The results in Figure 7 to Figure 10 show that the refueling time increases steadily as the thermal conductivity is reduced. These specific results are shown for a cylindrical pellet with 1 $\mathrm{cm}$ diameter and $1 \mathrm{~cm}$ height; these results remain qualitatively consistent for other pellet sizes 
and refueling pressure as well. Figure 7 shows the volume averaged temperature, amount of hydrogen stored (absolute $\mathrm{kg}$ of hydrogen per $\mathrm{kg}$ of pellet), and the amount of hydrogen stored per $\mathrm{m}^{3}$ of the pellet. The latter includes the amount of hydrogen adsorbed as well as the hydrogen gas present in the void volume (i.e., equal to $\rho_{P} q+\rho_{g} \varepsilon_{g}$ ). The maximum amount of hydrogen stored at 30 bar and $80 \mathrm{~K}$ is $28.1 \mathrm{~kg} / \mathrm{m}^{3}$ for a pellet with $10 \% \mathrm{ENG}$ and $29.2 \mathrm{~kg} / \mathrm{m}^{3}$ for a pellet with $5 \%$ ENG. We consider a value of $95 \%$ of the total volumetric capacity as an arbitrary, though reasonable, target for comparing the refueling behavior of a single pellet. The time taken to reach this value is 17.0 seconds (10\% ENG), 31.2 seconds (intermediate) and 62.5 seconds ( $5 \% \mathrm{ENG}$ ). One could therefore reasonably conclude that a bed comprised of 1 $\mathrm{cm} \times 1 \mathrm{~cm}$ pellets with $5 \% \mathrm{ENG}$ is unlikely to meet the refueling target of 3.3 minutes.

Figure 8 shows the temperature contours for the three cases at 2 and 5 seconds, whereas Figure 9 shows the temperature profiles at 25 seconds in a completely refueled pellet $(10 \%$ ENG), pellet nearing refueling (intermediate) and the pellet where refueling is still in progress (5\% ENG). It is clear that the qualitative behavior of pellet refueling is not changed: there is an initial surge in temperature, followed by a relatively gradual cooling of the pellet. The initial surge is attributed to the rapid pressurization of the pellet. Recall that the pellet reaches the equilibrium pressure of 30 bar within 0.1 seconds. Thus, the pressure-work done, reflected through the $\varepsilon_{g} \alpha_{\mathrm{gP}} T\left(\frac{\partial P}{\partial t}\right)$ term of energy balance Eq. (5), is a major contributor to the initial temperature surge. Additionally, it is clear from Figure $7(\mathrm{~b})$ that the amount of hydrogen adsorbed increases almost instantly from 0.008 to $0.02 \mathrm{~kg} / \mathrm{kg}$ adsorbent. Thus, the heat released on adsorption of hydrogen during rapid pressurization is another important contributor. It should be noted here that the actual amount of hydrogen adsorbed $(q)$ closely follows the equilibrium value $\left(q^{*}\right)$ for the entire range of conditions investigated.

The strong dependence of refueling time on the pellet thermal conductivity indicates that the refueling process is governed by the net thermal dynamics. The amount of heat that needs to be taken out depends on: (i) initial and final pellet temperatures, (ii) heat released on adsorption of hydrogen during the refueling process, and (iii) pressure work done. The former two are state functions and are independent of the path followed during refueling. The contribution of the latter is also similar for all the cases considered due to rapid pressurization. Since the velocity in the pellet is dependent on pressure gradients, the net transfer of heat due to bulk flow is negligible except for the initial $\sim 0.1$ to 0.2 seconds. Consequently, heat conduction through the pellet is the primary mode of heat transfer during the refueling process. Hence, we observe a strong dependence of refueling on the pellet thermal conductivity. 


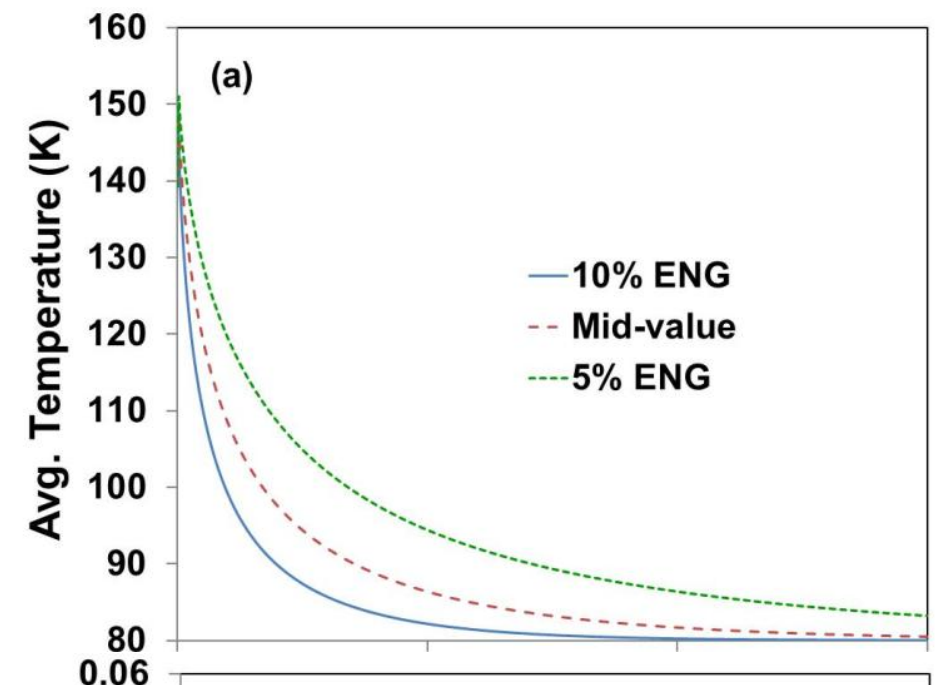

(b)
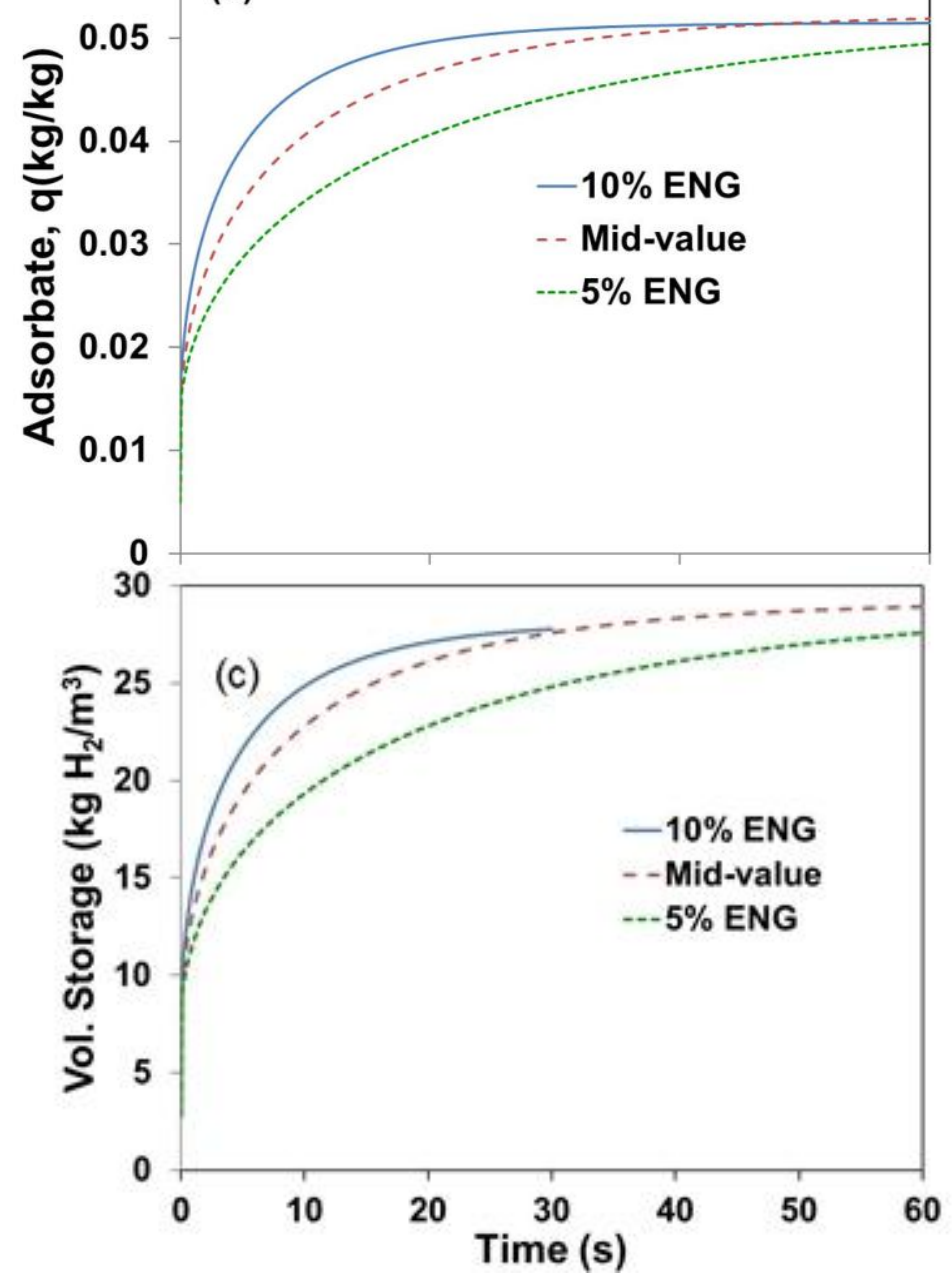

Figure 7: Transient evolution for three different values of thermal conductivity of (a) volume averaged pellet temperature, (b) amount of hydrogen adsorbed, and (c) net volumetric storage of hydrogen for a $1 \mathrm{~cm} \times 1 \mathrm{~cm}$ pellet. The higher the thermal conductivity, the faster is the refueling time. 

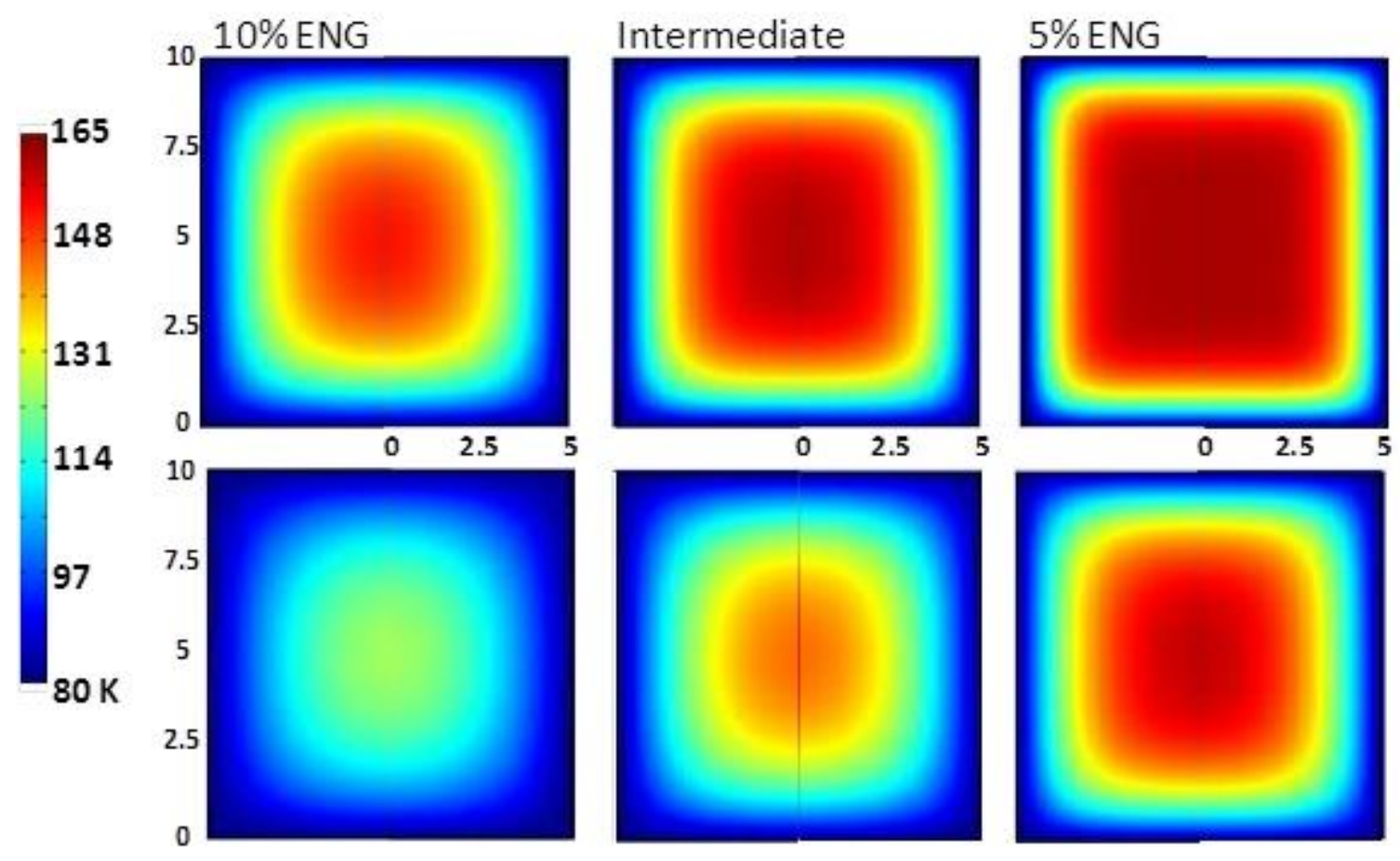

Figure 8: Temperature contours in the pellets for three different values of thermal conductivity at 2 seconds (top panels) and 5 seconds (bottom panels). The pellets cool uniformly in both radial and axial directions, though the rate of cooling is significantly higher for pellets with 10\% ENG.


Figure 9: Temperature contours for three different thermal conductivity values at 25 seconds. Refueling is completed for the first case, is nearing completion for the second case, whereas significant temperature gradients exist for MOF-5 with $5 \%$ ENG. 


\subsection{ANISOTROPIC THERMAL CONDUCTIVITY}

The work discussed so far used a scalar value for thermal conductivity of the pellet. However, new experimental data indicates that the in-plane value of thermal conductivity can be five times greater than the axial thermal conductivity. This anisotropy is due to the peculiar alignment of ENG in compacted pellets. ENG have a worm-like structure and they align in-plane during compaction, leading to enhanced thermal conductivity in the radial direction than axial. In this section, we use $\lambda_{e f f}(T, P)$ to refer to the polynomial function for the $10 \%$ ENG case from Table 2, and $\lambda_{\text {rad }}$ and $\lambda_{a x}$ to refer to axial and radial thermal conductivity values, respectively.

Figure 10 shows the effect of radial thermal conductivity on pellet refueling. The axial conductivity is kept at the nominal value of $\lambda_{a x}=\lambda_{e f f}(T, P)$, while two cases of $\lambda_{\text {rad }}=\{2,4\} \times$ $\lambda_{e f f}$ are considered. Increasing the radial thermal conductivity decreases the refueling time from 17.0 to 10.0 and 5.6 seconds, respectively. Figure 11 shows the temperature contours at $0.5,2$ and 5 seconds for $\lambda_{\text {rad }}=4 \times \lambda_{\text {eff }}(T, P)$. As expected, the rate of heat transfer is higher in the radial direction than the axial direction. In the isotropic case (for example, see Figure 3 bottom panels) where heat loss was uniform in radial and axial directions, the axisymmetric projections of temperature contours were approximately circular in shape; the temperature contours in Figure 11 are elongated since the heat is transferred faster in the radial direction.

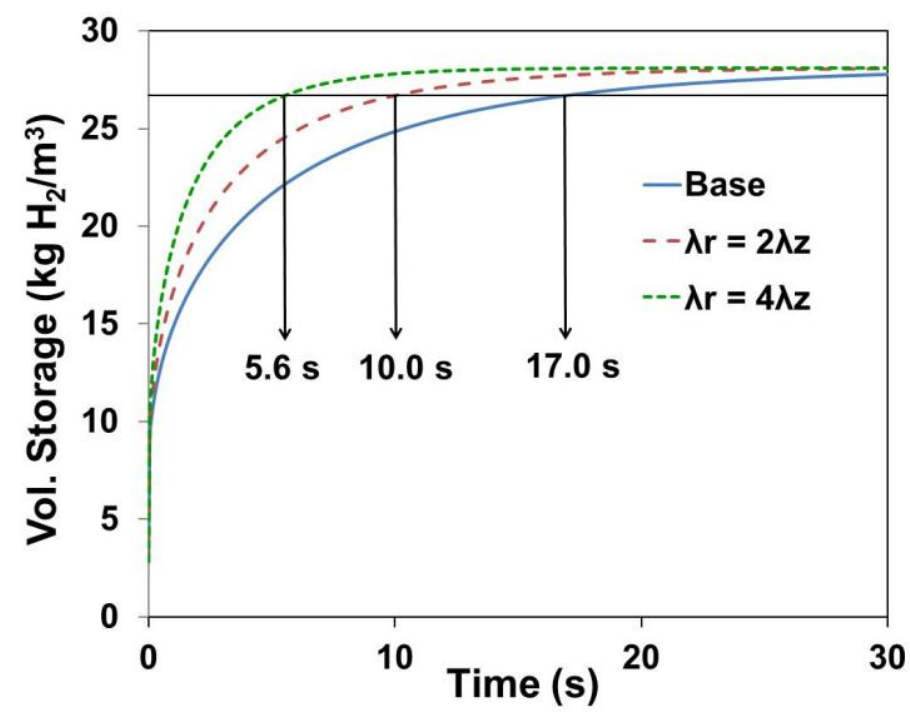

Figure 10: Effect of anisotropic thermal conductivity on pellet refueling. The solid line corresponds to $\lambda_{\text {rad }}=\lambda_{a x}$; the axial thermal conductivity is kept constant and the radial value is increased in the two dashed lines.

To further understand the role of thermal conductivity, a sensitivity analysis is done by 
varying $\lambda_{a x}$ for a constant $\lambda_{\text {rad }}$, and vice versa. The three cases of $\left\{\lambda_{\text {rad }}=\lambda_{e f f}(T, P) ; \lambda_{a x}=\right.$ $\left.\lambda_{e f f}(T, P)\right\},\left\{4 \times \lambda_{e f f} ; \lambda_{e f f}\right\}$ and $\left\{\lambda_{e f f} ; 4 \times \lambda_{e f f}\right\}$ are compared in Figure 12. The baseline case takes the maximum time to reach steady state, the case with higher $\lambda_{\text {rad }}$ takes minimum time whereas the time to steady state is intermediate when $\lambda_{a x}$ is increased. This is also reflected in the fact that the refueling is slowest for the baseline case (17.0 seconds), is faster when $\lambda_{a x}$ is increased at constant $\lambda_{\text {rad }}\left(9.0\right.$ seconds) and the fastest when $\lambda_{\text {rad }}$ is increased at constant $\lambda_{a x}$ (5.6 seconds). This shows that $\lambda_{\text {rad }}$ affects pellet refueling more strongly than $\lambda_{a x}$. This result is expected because the surface area of the pellet for axial heat transfer exposed to the surrounding cryogenic hydrogen is $(\pi / 2) d^{2}$ (two faces of the cylinder), while the surface area exposed for radial heat transfer is $\pi d^{2}$ (circumferential area, when $h=d$ ).

In summary, higher thermal conductivity results in faster refueling and lower temperature surge in the pellet. The radial component of thermal conductivity has a comparatively greater effect on refueling than the axial component.

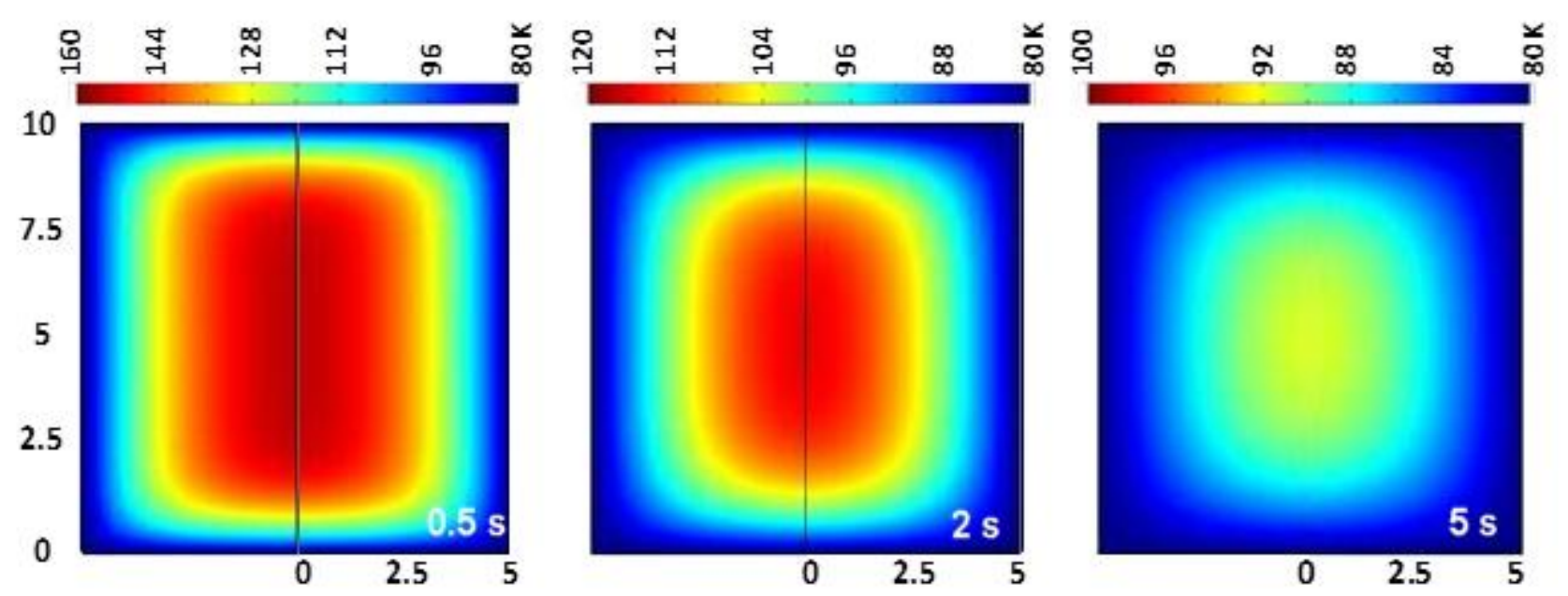

Figure 11: Temperature contours at various times for the case: $\lambda_{\text {rad }}=4 \lambda_{\text {ax }}$. 


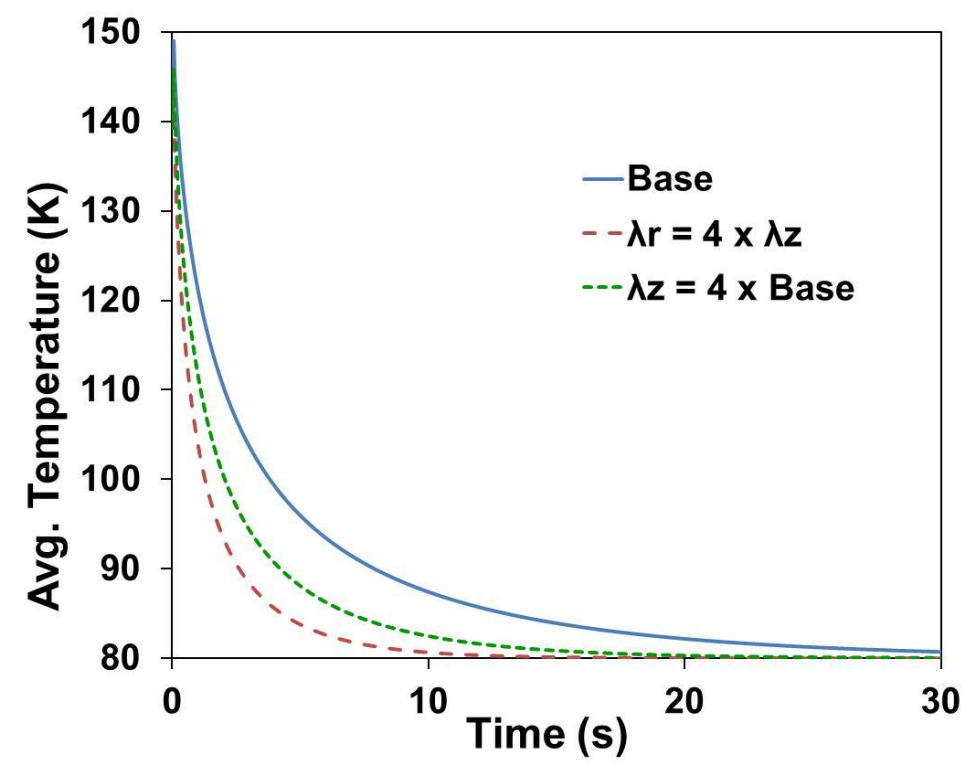

Figure 12: A sensitivity study for anisotropic thermal conductivity with $\lambda_{\text {rad }}=4 \lambda_{a x}$ and $\lambda_{a x}=4 \lambda_{\text {rad }}$. The lower of the two values is kept at the baseline.

\section{OPTIMAL PELLET SIZING}

The previous section analyzed the effect of pellet properties on refueling behavior. The question of choosing the optimal pellet size for fast refueling is considered in this section. For this purpose, the pellet with anisotropic thermal conductivity, $\lambda_{\text {rad }}=4 \times \lambda_{e f f}(T, P)$ and $\lambda_{a x}=$ $\lambda_{e f f}(T, P)$ is considered.

The pellet size effects with one of the dimensions kept constant are shown in Figure 13(a) and (b). In the former, pellet diameter is kept constant at $1 \mathrm{~cm}$ and the pellet height is varied; in the latter, pellet diameter is varied at a constant pellet height of $1 \mathrm{~cm}$. The solid lines in both of the figures represent the baseline case, dashed lines represent higher aspect ratios $(h / d>1$, i.e., "stick-like" pellets) and the dash-dot lines represent lower aspect ratios $(h / d<1$, i.e., "hockey-puck" pellets). Based on the results of the previous section, one can expect that increasing the circumferential surface area is likely to have a greater effect on the pellet refueling behavior. Thus, when the pellet height is varied at a constant diameter, both stick and hockey-puck pellets show acceptable refueling behavior that is likely to meet the overall refueling targets. When the pellet height is reduced, the volume also reduces, thus resulting in faster refueling (compare the dash-dot lines in Figure 13a). When the pellet height is increased to $2 \mathrm{~cm}$, the refueling time increases slightly; on further increasing the pellet height to $5 \mathrm{~cm}$, the effect on refueling behavior is marginal. The refueling time is not affected significantly when the 
pellet aspect ratio is increased beyond $h / d \approx 2$. This is because at very high aspect ratios, the primary heat transfer is primarily through the circumferential surface of the pellet. On the other hand, while refueling times are faster for hockey-puck pellets of height $1 \mathrm{~cm}$, a hockey-puck pellet with $\{h, d\}=\{1,2\} \mathrm{cm}$ is unlikely to meet the refueling targets (see Figure $13 \mathrm{~b}$ ).



Figure 13: Effect of varying (a) pellet height at constant diameter of $1 \mathrm{~cm}$, and (b) pellet diameter at constant height of $1 \mathrm{~cm}$ on refueling time. Numbers in brackets represent the height and diameter of the pellet, respectively.

An alternative method for optimizing the pellet size is to compare the refueling behavior for varying aspect ratios at a constant volume. The refueling time, defined as the time taken to reach $95 \%$ of the volumetric storage capacity (i.e., to reach $26.7 \mathrm{~kg}-\mathrm{H}_{2} / \mathrm{m}^{3}$-pellet) is plotted vs. aspect ratio for a constant volume of $\pi / 4 \mathrm{~cm}^{3}$ in Figure 14. The vertical line indicates the nominal aspect ratio 1.0 (with $h=d=1 \mathrm{~cm}$ ). The refueling time is highest for aspect ratio $h / d \approx 0.5$, i.e. when the diameter of the pellet is twice its height. The refueling time decreases rapidly for stick-like pellets. Figure 16 shows that hockey-puck pellets with small pellet heights also give low refueling times. For example, the refueling time for the hockey-puck pellet $\{h=2.15 \mathrm{~mm}, d=21.5 \mathrm{~mm}\}$ (i.e., aspect ratio 0.1 ) is nearly the same as that for a stick-like pellet $\{h=25.2 \mathrm{~mm}, d=6.3 \mathrm{~mm}\}$ (i.e., aspect ratio 4.0). A single pellet with stick-like geometry may be manufactured by an extrusion process, or multiple pellets can be stacked one-abovethe-other to create a stick-like geometry. Alternatively, a bed made with thin disk-like pellets $(h / d<0.1)$ are also likely to meet the refueling targets. However, such a geometry may suffer from large pressure drops and, possibly, poor structural strength.

Finally, in order to aid choosing appropriate sized pellet, a 2-D contour plot for refueling time as a function of pellet height and diameter is shown in Figure 15. The shaded region 
indicates pellet sizes that yield a refueling time of 20 seconds. The value of 20 seconds is arbitrarily chosen as $10 \%$ of the DOE target of 3.3 minutes as the system refueling time. It is clear than any "stick-like" pellet with diameter less than $16 \mathrm{~mm}$, or any "hockey-puck" pellet with height less than $6 \mathrm{~mm}$ is likely to meet the refueling time of 20 seconds.

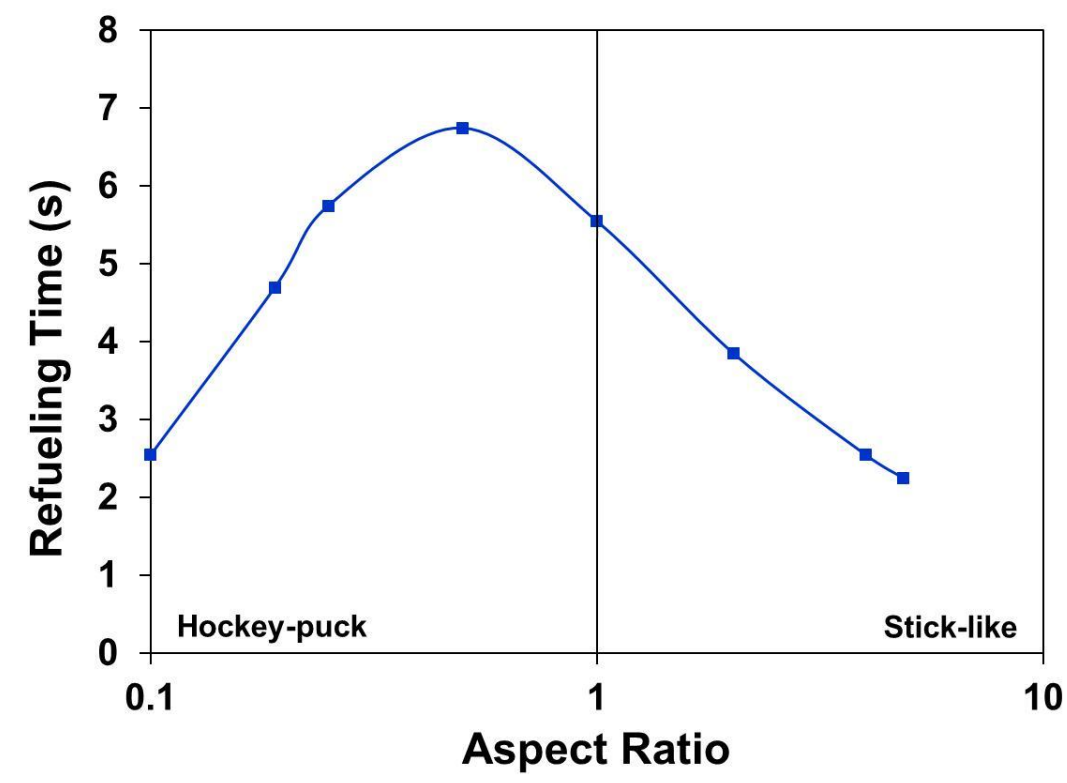

Figure 14: Time required for a pellet of volume $\pi / 4 \mathrm{~cm}^{3}$ to reach $95 \%$ of its volumetric storage capacity as a function of aspect ratio $(h / d)$. Both "stick-like" and "hockey-puck" pellets take less time to refuel compared to a pellet with $d=2 h$.

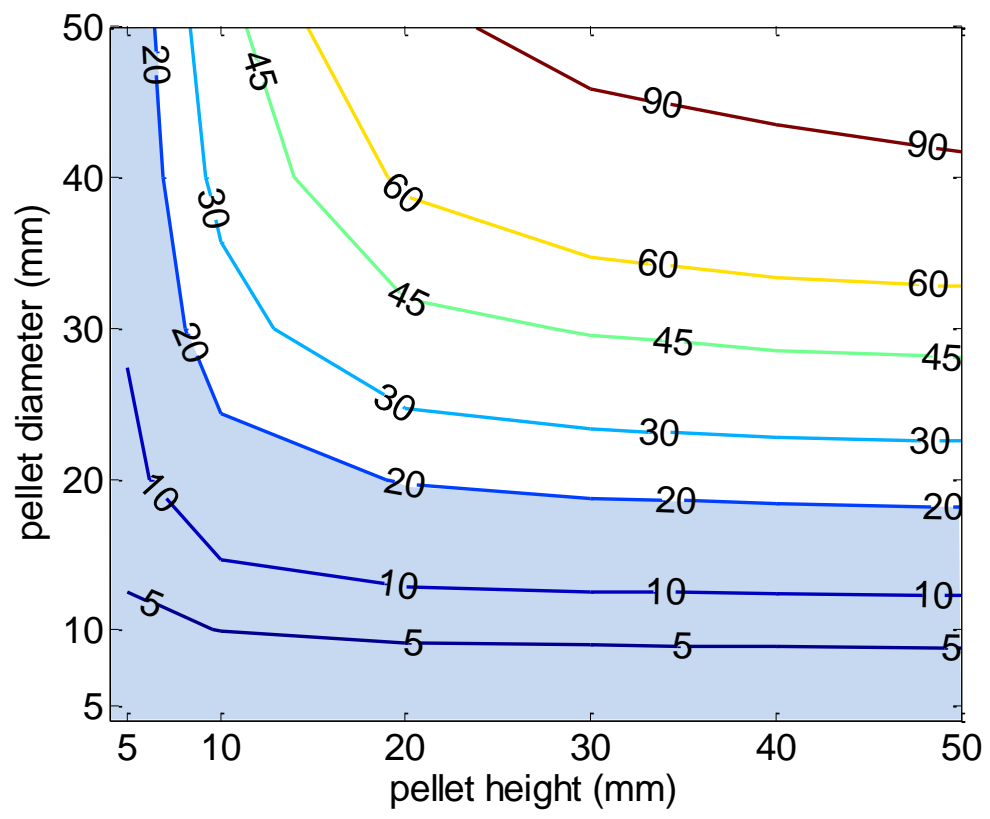

Figure 15: Contours of refueling time taken (in seconds) to reach $95 \%$ of the volumetric 
storage capacity. The shaded region indicates pellet sizes that yield refueling time less than 20 seconds.

\section{CONCLUSIONS}

The model for single pellet refueling was successfully ported to $\mathrm{COMSOL}^{\circledR} 4.2$ using an absolute-adsorption based isotherm. The results were benchmarked with an earlier excessadsorption based model. Refueling of a single pellet was found to proceed in two stages: an initial stage of rapid pressurization with a resulting increase in adsorbate concentration and temperature, followed by gradual cooling. The latter is primarily affected by the thermal dynamics of the pellet.

The roles of pellet permeability and thermal conductivity on single pellet refueling were investigated. Pellet permeability did not have a major effect on refueling behavior of a single pellet. The refueling behavior of a single pellet was found to be independent of the permeability for the range of permeability values considered here. Thermal conductivity, on the other hand, had profound impact on the rate of pellet cooling, and therefore the pellet refueling time.

The effect of anisotropic thermal conductivity was studied. Increasing the in-plane or radial thermal conductivity was found to have a greater impact on refueling than increasing the axial thermal conductivity by the same factor. Since the in-plane alignment of ENG enhances the radial thermal conductivity, heat transfer through the circumferential area of the cylindrical pellet is comparatively more efficient than heat transfer through the cylinder faces. Consequently, stick-like arrangement of pellets (which can then be stacked together in a "honey-comb" structure for the entire adsorbent bed) would be optimal. These conclusions may be even stronger due to the anisotropic nature of thermal conductivity.

Stick-like pellets with aspect ratios $h / d \gg 2$ were found to be the most suitable for achieving fast refueling times at a relatively high storage volume. Likewise, flat "hockey-puck" pellets are also likely to provide low refueling times. Conversely, short pellets, with $h / d \approx 0.5$ show the longest refueling times; a random packed bed of such pellets is not recommended based on this simulation study. Finally, Figure 15 provides a contour plot of refueling time as a function of pellet height and diameter, which may be used to choose the appropriate size of a single pellet. It can be concluded that the refueling of a single pellet is primarily governed by the adsorption isotherm and the pellet thermal conductivity.

\section{ACKNOWLEDGMENTS}

The authors acknowledge Senthil Kumar Vadivelu for his help in model formulation and 
implementation in $\mathrm{COMSOL}^{\circledR}$. Thanks are also due to Sudarshan Kumar for various discussions and for interfacing with the DOE Hydrogen Storage Engineering Center of Excellence partners for exchanging ideas and providing data. The efforts of Sudik and her group from Ford (permeability and isotherm data), and Marty Sulic (thermal conductivity data) are gratefully acknowledged. This paper was prepared as an account of work supported by the United States Department of Energy under Contract No. DE-FC36-09G019003. 


\section{REFERENCES}

1. Dunn, S. , "Hydrogen futures: Toward a sustainable energy system”, International Journal of Hydrogen Energy, 27 (2002) 235-264

2. Lemons, R.A. , "Fuel cells for transportation”, Journal of Power Sources, 29 (1990), 251264

3. Peighambardoust, S.J., Rowshanzamir, S., Amjadi, M., "Review of the proton exchange membranes for fuel cell applications", International Journal of Hydrogen Energy, 35 (2010), 9349-9384.

4. Hirscher, M. (2010) Handbook of Hydrogen Storage: New Materials for Future Energy Storage, Wiley $\mathrm{VCH}$

5. Schlapbach, L.; Zuttel, A., Hydrogen-storage materials for mobile applications. Nature 2001, 414, (6861), 353-358.

6. Yang, J.; Sudik, A.; Wolverton, C.; Siegel, D. J., High capacity hydrogen storage materials: Attributes for automotive applications and techniques for materials discovery. Chemical Society Reviews 2010, 39, (2), 656-675.

7. Petitpas, G., Bénard, P., Klebanoff, L.E., Xiao, J., Aceves, S., "A comparative analysis of the cryo-compression and cryo-adsorption hydrogen storage methods", International Journal of Hydrogen Energy, 39 (2014), 10564-10584.

8. Mori, D., Hirose, K., "Recent challenges of hydrogen storage technologies for fuel cell vehicles," International Journal of Hydrogen Energy, 34 (2009), 4569-4574

9. D.J. Durbin, C. Malardier-Jugroot, Review of hydrogen storage techniques for on board vehicle applications, International Journal of Hydrogen Energy, 2013, 38, 14595-14617

10. A P. Adametz, K. Müller, W. Arlt, Efficiency of low-temperature adsorptive hydrogen storage systems, International Journal of Hydrogen Energy 39 (2014) 15604-15613

11. R.K. Ahluwalia, J.K. Peng, Automotive hydrogen storage system using cryogenic adsorption on activated carbon, International Journal of Hydrogen Energy, 2009, 34, 5476-5487

12. Regli, L., Zecchina, A., Vitillo, J. G., Cocina, D., Spoto, G., Lamberti, C., Lillerud, K. P., Olsbye, U. and Bordiga, S., Hydrogen storage in Chabazite zeolite frameworks, Phys. Chem. Chem. Phys., 2005, 7, 3197-3203

13. M. P. Suh, H.J. Park, T.K. Prasad, and D.-W. Lim, Hydrogen Storage in Metal Organic Frameworks, Chemical Reviews 2012, 112, 782-835 
14. Rosi, N.L., Eckert, J., Eddaoudi, M., Vodak, D.T., Kim, J., O'Keeffe, M., Yaghi, O.M., "Hydrogen storage in microporous metal-organic frameworks", Science, 300 (2003), 1127-1129

15. FreedomCAR and Fuel Partnership, Targets for Onboard Hydrogen Storage Systems for Light-Duty Vehicles In Energy, US DoE., Ed. 2009; pp 9-10.

http://www1.eere.energy.gov/hydrogenandfuelcells/storage/pdfs/targets onboard hydro storage explanation.pdf (Last accessed: 18-April-2012) [Note: Revised link below] http://energy.gov/sites/prod/files/2014/03/f11/targets onboard hydro storage explanati on.pdf (Last accessed: 01-February-2015).

16. Kaye, S. S.; Dailly, A.; Yaghi, O. M.; Long, J. R., Impact of preparation and handling on the hydrogen storage properties of $\mathrm{Zn} 4 \mathrm{O}(1,4-$ benzenedicarboxylate)(3) (MOF-5). Journal of the American Chemical Society 2007, 129, (46), 14176-+.

17. Zhou, W.; Wu, H.; Hartman, M. R.; Yildirim, T., Hydrogen and methane adsorption in metal-organic frameworks: A high-pressure volumetric study. Journal of Physical Chemistry C 2007, 111, (44), 16131-16137.

18. Benard, P.; Chahine, R., Storage of hydrogen by physisorption on carbon and nanostructured materials. Scripta Materialia 2007, 56, (10), 803-808.

19. Marco-Lozar, J. P.; Juan-Juan, J.; Suarez-Garcia, F.; Cazorla-Amoros, D.; LinaresSolano, A., MOF-5 and activated carbons as adsorbents for gas storage. International Journal of Hydrogen Energy 2012, 37, (3), 2370-2381.

20. Hardy B., Corgnale C., Chahine R., Richard M-A., Garrison S., Tamburello D., Cossement D., and Anton D., Modeling of adsorbent based hydrogen storage systems, International Journal of Hydrogen Energy 2012, 37, 5691-5705

21. Purewal, J. J.; Liu, D.; Yang, J.; Sudik, A.; Siegel, D. J.; Maurer, S.; Muller, U., Increased volumetric hydrogen uptake of MOF-5 by powder densification. International Journal of Hydrogen Energy 2012, 37, (3), 2723-2727.

22. Liu, D.; Purewal, J. J.; Yang, J.; Sudik, A.; Maurer, S.; Mueller, U.; Ni, J.; Siegel, D. J., MOF-5 composites exhibiting improved thermal conductivity. International Journal of Hydrogen Energy 2012, 37, (7), 6109-6117.

23. Purewal, J.; Liu, D.; Sudik, A.; Veenstra, M.; Yang, J.; Maurer, S.; Muller, U.; Siegel, D. J., Improved hydrogen storage and thermal conductivity in high-density MOF-5 composites. Journal of Physical Chemistry 2012, 116, (38), 20199-20212. 
24. Sudik, A.; Veenstra, M.; Müller, U.; Maurer, S.; Gaab, M.; Purewal, J.; Liu, D. a.; Xu, C.; Siegel, D.; Ni, J., Adsorbent Materials for HSECoE, In Hydrogen Storage Engineering Technical Team Review, 2012.

25. Kumar, V. S.; Kumar, S., Generalized model development for a cryo-adsorber and 1-D results for the isobaric refueling period. International Journal of Hydrogen Energy 2010, 35, (8), 3598-3609.

26. Kumar, V. S., A generalized cryo-adsorber model and 2-D refueling results. International Journal of Hydrogen Energy 2011, 36, (23), 15239-15249.

27. Richard M-A., Bénard P. and Chahine R., Gas adsorption process in activated carbon over a wide temperature range above the critical point. Part 1: modified DubininAstakhov model, Adsorption, 2009, 15, 929-5607

28. R. Paggiaro, F. Michl, P. Bénard c, W. Polifke, Cryo-adsorptive hydrogen storage on activated carbon. II: Investigation of the thermal effects during filling at cryogenic temperatures, International Journal of Hydrogen Energy, 2010, 35, 648-659

29. J. Xiao, Tong L., Cossement D., Bénard P. and Chahine R., CFD simulation for chargedischarge cycle of cryo-adsorptive hydrogen storage on activated carbon, International Journal of Hydrogen Energy, 2012, 37, 12893-12904

30. J. Xiao, Hu M., Cossement D., Bénard P., and Chahine R., Finite element simulation for charge-discharge cycle, of cryo-adsorptive hydrogen storage on activated carbon, International Journal of Hydrogen Energy, 2012, 37, 12947-12959

31. Xiao, J. S.; Tong, L. A.; Deng, C. H.; Benard, P.; Chahine, R., Simulation of heat and mass transfer in activated carbon tank for hydrogen storage. International Journal of Hydrogen Energy 2010, 35, (15), 8106-8116.

32. J. Xiao, Li Q., Cossement D., Bénard P., and Chahine R., Lumped parameter simulation for charge-discharge cycle of cryo-adsorptive hydrogen storage system, International Journal of Hydrogen Energy, 2012, 37, 13400-13408

33. M. Raju, J. P. Ortmann, Sudarshan Kumar, System simulation model for high-pressure metal hydride hydrogen storage systems, International Journal of Hydrogen Energy, 2010, 35, 8742-8754

34. E. Dundar, Zacharia R., Chahine R. and Bénard P., Performance comparison of adsorption isotherm models for supercritical hydrogen sorption on MOFs, Fluid Phase Equilibria, 2014, 363, 74-85. 
35. Ming, Y., Purewal, J., Liu, D., Sudik, A., Xu, C., Yang, J., Veenstra, M., Rhodes, K., Soltis, R., Warner, J., Gaab, M., Müller, U., Siegel, D.J., "Thermophysical properties of MOF-5 powders", Microporous and Mesoporous Materials, 185 (2014), 235-244

36. Myers, A. L.; Monson, P. A., Adsorption in porous materials at high pressure: Theory and experiment. Langmuir 2002, 18, (26), 10261-10273.

37. Richard, M. A.; Benard, P.; Chahine, R., Gas adsorption process in activated carbon over a wide temperature range above the critical point. Part 2: Conservation of mass and energy. Adsorption-Journal of the International Adsorption Society 2009, 15, (1), 53-63.

38. Lemmon, E. W.; Huber, M. L.; McLinden, M. O. NIST Standard Reference Database 23: Reference Fluid Thermodynamic and Transport Properties-REFPROP, 9.0; National Institute of Standards and Technology: Gaithersburg, MD, USA, 2010.

39. Leachman, J. W.; Jacobsen, R. T.; Penoncello, S. G.; Lemmon, E. W., Fundamental Equations of State for Parahydrogen, Normal Hydrogen, and Orthohydrogen. Journal of Physical and Chemical Reference Data 2009, 38, (3), 721-748.

40. Kumar, D.; Chakraborty, A.; Kumar, S. V.; Cai, M., Adsorbent bed thermal management. In Hydrogen Storage Engineering Technical Team Review, 2012.

41. Sulic, M.; Kumar, D., Low temperature thermal conductivity of MOF-5 pellets. Personal Communication: July 2011. 